\title{
Photodynamic therapy for the eradication of biofilms formed by catheter associated Pseudomonas aeruginosa strains
}

\author{
Viviana Teresa Orlandi', Francesca Villa', Serena Cavallari'², Paola Barbieri', Stefano Banfi', \\ Enrico Caruso', Pierangelo Clerici² \\ I Università degli Studi dell'Insubria, Dipartimento di Biologia Strutturale e Funzionale - Varese \\ 2 Ospedale civile di Legnano, U.O. di Microbiologia - Legnano (MI)
}

Key words: Photodynamic therapy, Biofilm, Pseudomonas aeruginosa

Applicazione della terapia fotodinamica (PDT) a biofilm formati da ceppi di Pseudomonas aeruginosa isolati da cateteri urinari

\section{SUMMARY}

Pseudomonas aeruginosa has emerged as a major opportunistic pathogen causing catheter-associated urinary tract infections (CA-UTIs) associated with high mortality and morbidity. In this study 18 . aeruginosa isolates from urine of catheterized patients were evaluated for in vitro biofilm formation.All the tested strains showed the ability to form biofilm more thicker than those formed by a cohort of 29 blood culture strains belonging to the same species.

Photodynamic antimicrobial chemotherapy (PACT) is a novel antimicrobial treatment that exploits a photosensitizer (PS) and visible light to induce lethal oxidative damages in bacterial cells and could be used as local antimicrobial approach in CA-UTIs.

Here we tested the susceptibility of planktonic and sessile cultures of $P$. aeruginosa strains, the model strain PAOI and CA-UTI isolates, to photodynamic inactivation with a di cationic porphyrinic photosensitizer, the 5, I5-di (N-benzyl-4-pyridynium)-porphyrin di chloride. Although Pseudomonas aeruginosa is regarded as a difficult target for antimicrobial chemotherapy, satisfactory bactericidal activities on both planktonic and biofilm cultures were observed.

\section{INTRODUZIONE}

Le infezioni delle vie urinarie associate a catetere (CA-IVU) rappresentano una frazione rilevante delle infezioni nosocomiali che spesso causano patologie, sepsi e morte oltre a incidere notevolmente sulle spese del sistema sanitario (8). Il catetere urinario offre una superficie riconosciuta dalle adesine di uropatogeni favorendone la colonizzazione e inoltre, alterando la funzionalità dello svuotamento della vescica, fornisce un terreno adatto alla crescita microbica $(7,5)$. I primi batteri ad essere isolati sono Staphylococcus epidermidis, Escherichia coli ed Enterococcus faecalis. In un secondo momento compaiono Pseudomonas aeruginosa, Proteus mirabilis, Providencia stuartii, Morganella morganii e Klebsiella pneumoniae che sono più difficili da eradicare con gli antibiotici mentre il catetere è ancora in situ (9). In particolare è nota la capacità di $P$. aeruginosa di formare biofilm (6), proteggendo questo microrganismo dalle difese dell'ospite e dagli approcci chemioterapici; quindi rendendo la sua eradicazione ancora più difficile al punto da essere la causa principale di infezioni persistenti e ricorrenti delle vie urinarie (2). Inoltre $P$. aeruginosa è spesso coinvolto in una fase di consolidamento del biofilm che può determinare l'occlusione del catetere, causando pielonefrite e setticemia (9).

Negli ultimi anni si stanno sempre più indagando metodi alternativi all'approccio chemioterapico tradizionale per contrastare tali forme di aggregazione batterica. Recentemente è stata presa in considerazione l'applicazione della terapia fotodinamica (PDT) nei confronti di colture batteriche. La PDT, originariamente nata come terapia antitumorale e che sta ottenendo sempre più consensi in campo clinico, accoppia l'uso di fotosensibilizzanti (PS) e luce visibile determinando un danno ossidativo alle cellule. Quando il PS assorbe la radiazione luminosa passa da uno stato elettronico fondamentale ad uno eccitato quindi può trasferire la sua energia alle molecole adiacenti tra le quali è importante 1'ossigeno molecolare $\left({ }^{3} \mathrm{O}_{2}\right)$, che passa allo stato di singoletto eccitato $\left({ }^{1} \mathrm{O}_{2}\right)$, forma altamente reattiva e tossica. L'ossigeno sin- 
goletto danneggia la parete cellulare, determina l'inibizione della sintesi delle proteine e l'alterazione molecolare del DNA, causando così un effetto mortale per i batteri (1).

In questo studio dopo aver valutato la capacità di formare biofilm da parte di ceppi di $P$. aeruginosa isolati da urinocolture da catetere si è proceduto all'applicazione dell'approccio fotodinamico sia al microrganismo modello PAO1 che ad alcuni ceppi provenienti dalla coorte di uropatogeni.

\section{MATERIALI E METODI \\ Ceppi batterici}

P. aeruginosa PAO1 (10), ceppo modello per lo studio dei biofilm batterici. I ceppi di P. aerugino$s a$ isolati da urinocolture da catetere (UR) e da emocolture (SA) sono stati identificati mediante sistema automatizzato Vitek 2 (bioMérieux).

\section{Allestimento e quantificazione dei biofilm}

Gli inoculi sono stati effettuati stemperando una colonia isolata da coltura pura del ceppo in esame in terreno TSB a $37^{\circ} \mathrm{C}$ per 24 ore in statico. Il giorno seguente sono state allestite delle subcolture in TSB $\left(\mathrm{OD}_{620 \mathrm{~mm}}=0.1\right)$ che sono state aliquotate in multipiastre da 96 pozzetti di polistirene a fondo piatto e incubate in statico per $24 \mathrm{~h}$ a $37^{\circ} \mathrm{C}$. La crescita della popolazione in forma planctonica è stata valutata mediante metodo turbidimetrico $(\mathrm{OD}=620 \mathrm{~nm})$; mentre per quantificare la biomassa adesa al pozzetto è stata effettuata la colorazione con cristalvioletto (CV). Dopo 2 lavaggi con acqua deionizzata e colorazione con cristalvioletto (1\%) per 20 minuti, sono stati effettuati 3 lavaggi con acqua deionizzata quindi la biomassa adesa è stata risospesa in etanolo $96 \%$ per procedere con la lettura spettrofotometrica (OD $620 \mathrm{~nm})$. Il valore di biofilm index (BI) viene calcolato tramite la seguente formula (4):

Biofilm index $=\mathrm{OD}_{620 \mathrm{~mm}}$ biofilm $\mathrm{x}\left(\mathrm{OD}_{620 \mathrm{~mm}}\right.$ inoculo / OD $620 \mathrm{~nm}$ crescita)

\section{Fotosensibilizzante}

Il fotosensibilizzante impiegato in questo lavoro è stato sintetizzato nei nostri laboratori e si tratta della la 5.15-di (N-benzil-4-piridinio) porfirina di cloruro (Figura I), da questo momento indicata come SV28. Questa molecola è caratterizzata da un nucleo tetrapirrolico recante nelle posizioni 5 , 15 sostituenti piridinici che sono stati alchilati all'azoto con benzil cloruro (N-benzilati) per ottenere le cariche positive necessarie all'interazione con le cellule batteriche.

\section{Protocollo di fotoinattivazione}

Per le prove preliminari di fotoinattivazione i biofilm sono stati allestiti nel seguente modo: una coltura cresciuta $\mathrm{O} / \mathrm{N}$ a $37^{\circ} \mathrm{C}$ in $\mathrm{LB}$ viene diluita 1:100 in terreno minimo M9 (glucosio $5 \mathrm{mM}$ ) e aliquotata in una piastra da 12 pozzetti di polisti- rene. Dopo incubazione $\mathrm{O} / \mathrm{N}$ a $37^{\circ} \mathrm{C}$ viene somministrato il fotosensibilizzante (PS) alla concentrazione scelta. Dopo incubazione al buio per 60 minuti, la piastra viene sottoposta a irraggiamento mediante lampada tungsteno-alogena da 500 watt per $75 \mathrm{~min}\left(266 \mathrm{~J} / \mathrm{m}^{2}\right)$. La colorazione con cristalvioletto viene eseguita come descritto in precedenza.

Il trattamento fotodinamico è stato effettuato anche su cellule cresciute in forma planctonica ed usando concentrazioni variabili di PS comprese tra 1 e $20 \mu \mathrm{M}$; in questo caso l'efficacia di tale trattamento è stata valutata mediante conta vitale (cfu/ml) dei sopravviventi.

\section{Up-take del fotosensibilizzante}

Le colture sono state allestite nella stessa modalità degli esperimenti di fotoinattivazione. Le microprovette contenenti $1.5 \mathrm{ml}$ di coltura dopo essere state incubate per un'ora al buio a $37^{\circ} \mathrm{C}$ con il fotosensibilizzante alla concentrazione scelta. Di seguito i campioni sono stati centrifugati (13000 rpm per 10 minuti) in modo da allontanare il fotosensibilizzante che non ha interagito con le cellule. Il PS rimasto nel surnatante è stato successivamente quantificato tramite misure spettrofotometriche nell'intervallo di lunghezze d'onda 380-800 nm; per SV28 il picco di assorbimento nel terreno minerale utilizzato (M9) si registra a $410 \mathrm{~nm}$. Per dosare il PS legato alle cellule batteriche il pellet è stato risospeso in $500 \mu \mathrm{l}$ di SDS $2 \%$ e lasciato a temperatura ambiente per 30 minuti, quindi centrifugato (13000 rpm per 10 minuti) e il surnatante utilizzato per la quantificazione del PS; per SV28 il picco di assorbimento nel mezzo utilizzato (SDS) si registra a $420 \mathrm{~nm}$. Come controlli sono stati utilizzati campioni di cellule non trattate con il PS.

\section{Analisi statistica}

Le prove di formazione del biofilm da parte dei ceppi clinici sono state effettuate almeno in triplicato. Per ogni ceppo è stata calcolata la media dei valori di OD $_{620 \mathrm{~nm}}$ dopo colorazione con cristalvioletto e la corrispondente deviazione standard (SD). La significatività rispetto al ceppo di riferimento PAO1 è stata calcolata mediante test ANOVA a una via.

\section{RISULTATI}

Valutazione della produzione di biofilm da parte di ceppi di $P$. aeruginosa

Tutti i ceppi di Pseudomonas aeruginosa isolati da urinocolture da catetere e da emocolture considerati per questo studio hanno formato un biofilm su supporto di polistirene. La maggior parte dei ceppi ha formato anche una pellicola liquido-aria. Per valutare la propensione a formare biofilm di un microrganismo può essere utilizzato il biofilm 
index (BI) (4). Tale parametro permette di normalizzare la biomassa adesa sia alla quantità di cellule inoculate che alla frazione di cellule cresciute in sospensione. Nel protocollo adottato si è standardizzata la procedura in modo da utilizzare gli inoculi ad una $\mathrm{OD}_{620 \mathrm{~nm}} \sim 0.1$. A parità di inoculo, più il $\mathrm{BI}$ è elevato e più il ceppo predilige la forma adesa rispetto a quella planctonica. Il BI è stato calcolato effettuando la colorazione senza allontanare la pellicola liquido/aria, rappresentando anche quest'ultima una forma di biofilm batterico. In particolare il 31\% dei ceppi da emocolture ( 9 su 29) ha mostrato valori di BI statisticamente superiori a quelli di PAO1, mentre solo 2 ceppi su 18 ceppi urinari hanno mostrato un valore statisticamente superiore al BI di PAO1 (0.18).

Per valutare la quantità di biofilm prodotto è stata eseguita una colorazione con cristalvioletto che permette una valutazione semiquantitativa della biomassa adesa al supporto, dopo rimozione meccanica della pellicola liquido-aria e della parte liquida. Nelle condizioni adottate in questo lavoro (crescita in statico) 7 su 18 ceppi urinari hanno formato un biofilm significativamente più abbondante rispetto a quello del microrganismo modello PAO1 (Tabella 1): UR02 ( $\mathrm{p}=0.038), \mathrm{UR} 03$ $\left(\mathrm{p}=1.1 \times 10^{-6}\right), \operatorname{UR} 04(\mathrm{p}=0.006), \operatorname{UR} 10(\mathrm{p}=0.011)$, UR21 $\left(\mathrm{p}=1.9 \mathrm{x} 10^{-4}\right), \mathrm{UR} 44\left(\mathrm{p}=1.6 \times 10^{-4}\right)$ e UR47 $(\mathrm{p}=0.006)$. Per i ceppi urinari la media dei valori di $\mathrm{OD}_{620 \mathrm{~mm}}$ dopo colorazione con cristalvioletto è risultata pari a $0.4 \pm 0.39$ maggiore del valore medio ottenuto con $P$. aeruginosa PAO1 ( $0.34 \pm$ 0.11) (Tabella 1).

Tra i 29 ceppi di $P$. aeruginosa isolati da emocolture solo il ceppo SA23 ha formato un biofilm più abbondante rispetto al microrganismo modello $\left(\mathrm{p}=5.4 \times 10^{-4}\right)$. In questa coorte la media dei valori di $\mathrm{OD}_{620 \mathrm{~nm}}$ è risultata pari a $0.17 \pm 0.05$, inferiore a quella di PAO1 $(0.34 \pm 0.11)$ e dei ceppi isolati da catetere $(0.4 \pm 0.39)$. I ceppi isolati da urine da catetere hanno quindi mostrato una capacità di formare biofilm significativamente più abbondanti rispetto a quelli formati dai ceppi isolati da campioni di sangue.

\section{Applicazione della terapia fotodinamica a $P$. aeruginosa PAO1}

Il protocollo di fotoinattivazione è stato applicato alla coltura planctonica di Pseudomonas aeruginosa PAO1 utilizzando come fotosensibilizzante la di-arilporfirina di-cationica SV28. Dopo l'incubazione al buio necessaria per far interagire il PS con la parete del microrganismo segue la fase di illuminazione che determina la formazione dei prodotti radicalici derivati dall'ossigeno molecolare che danneggiano le componenti cellulari. SV28 non ha mostrato tossicità intrinseca nei con- fronti di P. aeruginosa (Figura II): la concentrazione cellulare dopo trattamento al buio della coltura rimane inalterata all'aumentare della concentrazione della porfirina. La fotoinattivazione è invece un processo dose-dipendente: il PS attivato dalla luce, alla concentrazione di $1 \mu \mathrm{M}$ e 2.5 $\mu \mathrm{M}$, non presenta fototossicità, mentre a $5 \mu \mathrm{M}$ e $10 \mu \mathrm{M}$ la molecola SV28 esplica la sua attività fototossica su PAO1 determinando rispettivamente una diminuzione del titolo di tre e quattro unità logaritmiche. Aumentando la concentrazione di SV28 fino a $50 \mu \mathrm{M}$ si è raggiunto il massimo livello di fotoinattivazione rilevabile con il sistema adottato, ma si è osservata anche una tossicità intrinseca con un abbattimento della popolazione di 2 unità logaritmiche (dato non mostrato).

Sono state effettuate delle prove preliminari volte a valutare la quantità di fotosensibilizzante accumulata dalle cellule intatte di $P$. aeruginosa PAO1. Per queste prove sono state utilizzate le condizioni e le concentrazioni di PS utilizzate negli esperimenti di fotoinattivazione illustrati in precedenza. Come si può osservare dalla Figura III, il PS non viene rilevato nella frazione non legata alla cellula (surnatante) ma si trova associato alla frazione cellulare in maniera dose dipendente. Ulteriori indagini mediante frazionamento delle componenti parietali potrebbero chiarire se la parete del batterio Gram negativo potrebbe essere sede dell'accumulo del PS.

\section{Applicazione della terapia fotodinamica ad alcuni ceppi clinici isolati da urine da catetere}

Test preliminari di fotoinattivazione sono stati condotti su alcuni ceppi, cresciuti in forma di biofilm, scelti tra quelli isolati da urine da catetere. Il ceppo di $P$. aeruginosa PAO1 è stato utilizzato come microrganismo modello. I biofilm sono stati incubati al buio per $1 \mathrm{~h}$ con la di-arilporfirina SV28 e irraggiati. Dei sei ceppi testati, ben 5 ceppi (PAO1, UR 4, UR7, UR 47 e UR 44) mostrano una evidente diminuzione della colorazione con cristalvioletto dopo incubazione col PS e irraggiamento, compatibile con una rimozione della biomassa adesa conseguente alla morte cellulare (Figura IV).

\section{DISCUSSIONE}

Valutando il Biofilm Index, sia i ceppi isolati da campioni di sangue e responsabili di sepsi che $\mathrm{i}$ ceppi isolati da urinocolture da catetere hanno mostrato una capacità di crescere sotto forma di biofilm. In particolare i ceppi isolati dal sangue, pur non formando biofilm abbondanti, hanno mostrato, rispetto ai ceppi urinari, una maggior propensione a crescere adesi piuttosto che in sospensione. 
Diversamente, i ceppi di Pseudomonas aerugino$s a$ isolati da urinocolure da catetere hanno mostrato una capacità di formare un biofilm più abbondante rispetto ai ceppi isolati da emocolture. I batteri cresciuti in questa forma risultano più tolleranti ai trattamenti chemioterapici e quindi più difficili da eradicare. Il catetere può infatti costituire un supporto molto adatto per i ceppi dotati di adesine, e tra i diversi uropatogeni $P$. aeruginosa è il più versatile e può crescere anche su cateteri in silicone che sono normalmente considerati meno soggetti a colonizzazione batterica rispetto a quelli di gomma e lattice (3). I ceppi invece isolati dalle emocolture formano biofilm meno abbondanti rispetto al microrganismo modello e ai ceppi urinari. La capacità di aderire al primo sito di colonizzazione, prima di essere immessi nel flusso ematico, potrebbe favorire la loro disseminazione.

Tra le strategie messe a punto per abbattere l'incidenza da batteriurie correlate a cateterizzazione a lungo e a breve termine, vi è l'incorporazione di sostanze biocide (ossido di argento, oro, palladio) o di antibiotici nel materiale del catetere, o anche lo sviluppo di materiali con caratteristiche della superficie in grado di prevenire l'adesione delle cellule batteriche (3). La terapia fotodinamica, inizialmente nata come applicazione antitumorale, negli ultimi anni sembra essere potenzialmente applicabile sia alle specie multiresistenti sempre più diffuse in ambito nosocomiale che ai biofilm batterici. Dalla letteratura è noto che i PS carichi positivamente sono più efficaci di quelli neutri e anionici, e che i batteri Gram positivi sono più sensibili ai fotosensibilizzanti cationici rispetto ai batteri Gram negativi, in quanto questi ultimi presentano una membrana esterna che potrebbe costituire un ostacolo all'ingresso del PS. Tra i microrganismi indagati, $P$. aeruginosa risulta essere una tra le specie più difficilmente debellabile con trattamento fotochimico. In questo lavoro si è utilizzato la molecola porfirinica dicationica (SV28) su ceppi clinici di $P$. aeruginosa: con il microrganismo modello PAO1 non si è raggiunto il massimo livello di fotoinattivazione rilevabile, limite che è stato invece raggiunto con analoghi trattamenti su $E$. coli (dati non mostrati). Poiché anche nel caso di $P$. aeruginosa il PS si lega in maniera dose-dipendente alla cellula e la frazione non legata è pari a zero: la non completa efficacia non sembra dovuta quindi ad una incapacità di interazione con la struttura cellulare.

Quando alcuni ceppi urinari sono stati sottoposti a fotoinattivazione mediante SV28, la colorazione con cristalvioletto ha messo in evidenza una chiara diminuzione della biomassa adesa. Questi dati preliminari evidenziano la potenzialità dell'approccio fotodinamico per trattamenti locali dei cateteri urinari che sono raggiungibili dalla sorgente luminosa, in accordo con quanto osservato da Wainwright che utilizzando PS commerciali quali il Blu di metilene e Blu di toluidina, ha ottenuto una migliore efficacia battericida nei confronti di ceppi CA-UTI rispetto a trimetoprim e levofloxacina (11).

Tabella I. Valori di densità ottica a $620 \mathrm{~nm}$ dopo colorazione con cristalvioletto.

\begin{tabular}{cc}
\hline CEPPO & OD $_{620 \mathrm{~nm}} \mathbf{C . V} \pm$ s.d. \\
\hline UR 0 I & $0.087 \pm 0.01$ \\
\hline UR 02 & $0.05 \pm 0.009\left(^{*}\right)$ \\
\hline UR 03 & $0.96 \pm 0.05\left(^{* *}\right)$ \\
\hline UR 04 & $1.41 \pm 0.09\left(^{* *}\right)$ \\
\hline UR 05 & $0.38 \pm 0.08$ \\
\hline UR 06 & $0.16 \pm 0.03$ \\
\hline UR 07 & $0.05 \pm 0.01$ \\
\hline UR 10 & $0.72 \pm 0.32\left(^{*}\right)$ \\
\hline UR 21 & $0.75 \pm 0.13\left(^{* *}\right)$ \\
\hline UR 39 & $0.06 \pm 0.01$ \\
\hline UR 4I & $0.21 \pm 0.06$ \\
\hline UR 42 & $0.02 \pm 0.01$ \\
\hline UR 43 & $0.10 \pm 0.01$ \\
\hline UR 44 & $0.92 \pm 0.24\left(^{* *}\right)$ \\
\hline UR 45 & $0.09 \pm 0.01$ \\
\hline UR 46 & $0.16 \pm 0.04$ \\
\hline UR 47 & $0.48 \pm 0.18$ \\
\hline UR 48 & $0.52 \pm 0.15\left(^{* *}\right)$ \\
\hline PAOI & $0.34 \pm 0.11$ \\
\hline
\end{tabular}

I valori sono espressi come medie di almeno 4 pozzetti \pm deviazione standard (sd). I ceppi di P. aeruginosa sono stati isolati da urinocolture da catetere. Tra parentesi la significatività rispetto al ceppo di riferimento PAOI calcolata mediante test ANOVA a una via $\left(* \mathrm{P}<0.05,{ }^{* * P}<0.01\right)$.

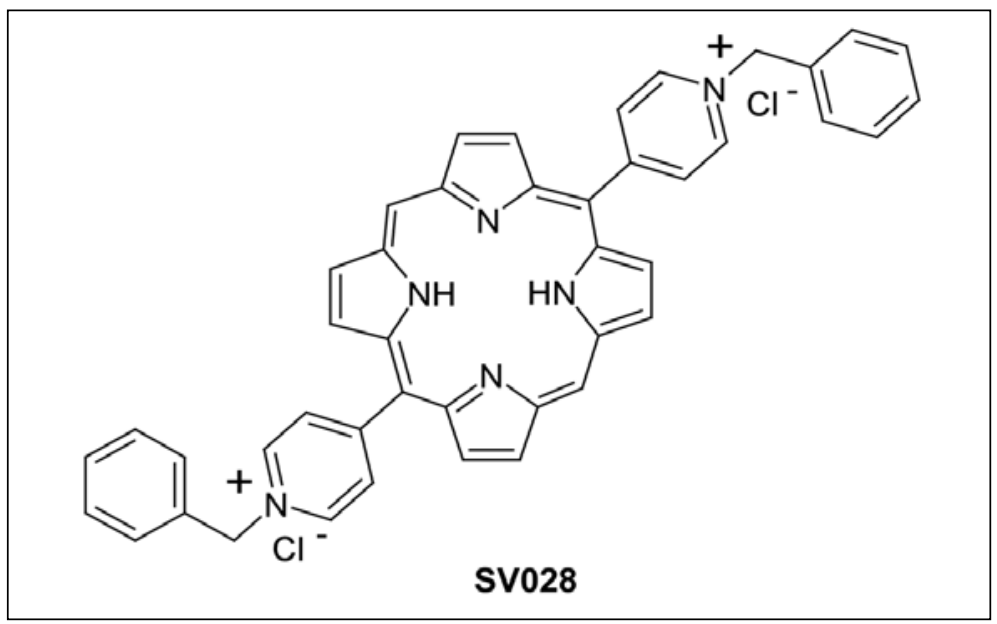

Figura I. SV28: 5.I5-di (N-benzil-4-piridinio) porfirina di cloruro. 


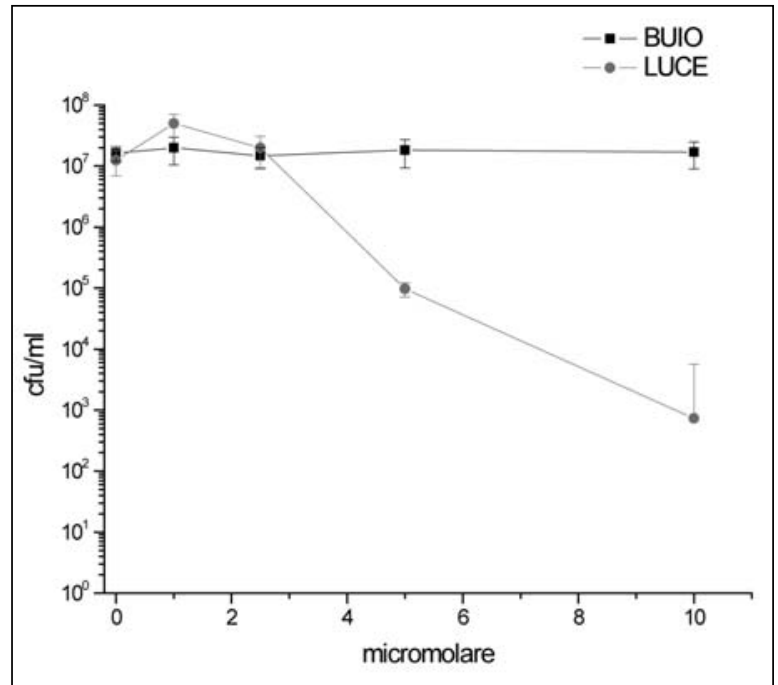

Figura II. Effetto dose dipendente di SV28 sulla vitalità di colture planctoniche di P. aeruginosa PAOI dopo incubazione al buio $(\mathrm{Ih})$ e dopo irraggiamento con lampada alogena $\left(266 \mathrm{~J} / \mathrm{m}^{2}\right)$.

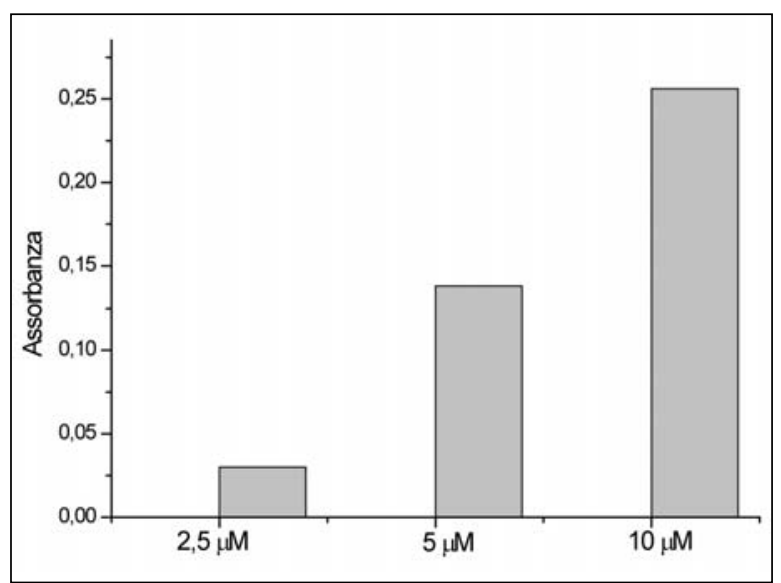

Figura III. Capacità di SV28 di legarsi alle cellule di P. aeruginosa PAOI in forma planctonica dopo incubazione al buio (Ih).

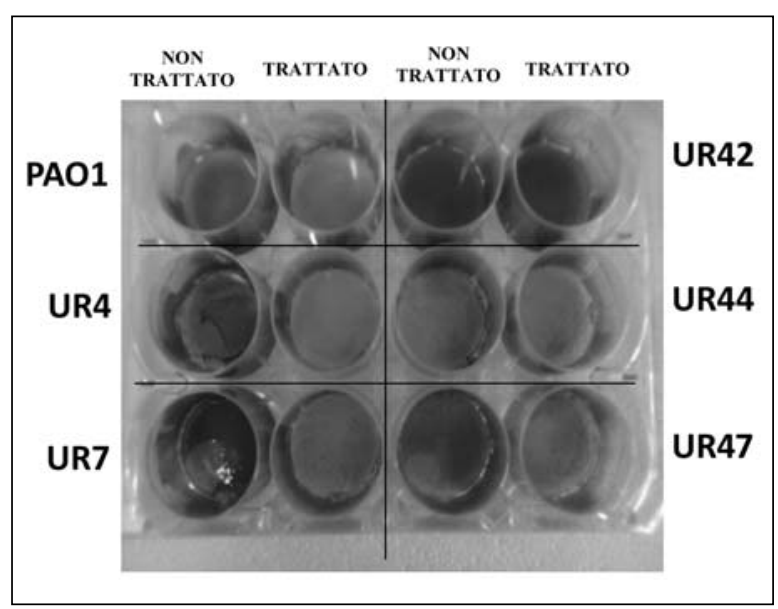

Figura IV. Riduzione della biomassa adesa, valutata mediante colorazione con cristal violetto, dopo trattamento fotodinamico di P. aeruginosa PAOI e di ceppi isolati da cateteri urinari.

\section{BIBLIOGRAFIA}

1. Calin MA, Parasca SV. Light sources for photodynamic inactivation of bacteria. Lasers Med Sci 2009; 24: 453-60.

2. Costerton JW, Stewart PS, Greenberg EP. Bacterial biofilms: a common cause of persistent infections. Science 1999; 284: 1318-22.

3. Espsosito S, Noviello S, Leone S. Le infezioni urinary associate a catetere: epidemiologia e prevenzione. Le infezioni in medicina 2008; 3: 130-43.

4. Fabretti F, Theilacker C, Baldassarri L, et al. Alanine esters of enterococcal lipoteichoic acid play a role in biofilm formation and resistance to antimicrobial peptides. Infect Immun 2006; 74(7): 4164-71.

5. Hashmi S, Kelly E, Rogers SO, Gates J. Urinary tract infection in surgical patients. Am J Surg 2003; 186: 53-6.

6. Jarvis WR, Martone WJ. Predominant pathogens in hospital infections. J Antimicrob Chemoth 1992; 29: 19-24.

7. Leone M, Garnier F, Avidan M, Martin M. Catheterassociated urinary tract infections in intensive care units. Microbes Infect 2004; 6: 1026-32.

8. Mittal R, Sharma S, Chibber S, Aggarwal S, Gupta V, Harjai $\mathrm{K}$. Correlation between serogroup, in vitro biofilm formation and elaboration of virulence factors by uropathogenic Pseudomonas aeruginosa. FEMS Immunol Med Microbiol 2010; 58: 237-43.

9. Stickler DJ. Medscape. Bacterial biofilms in patients with indwelling urinary catheters. Nat Clin Pract Urol 2008; 5(11): 598-608.

10. Stover CK, Pham XQ, Erwin AL, et al. Complete genome sequence of Pseudomonas aeruginosa PAO1, an opportunistic pathogen. Nature 2000; 406: 959-64.

11. Wainwright M, Stanforth A, Jones R, Loughran C, Meegan K. Photoantimicrobials as a potential local approach to geriatric UTIs. Lett Appl Microbiol 2010; 50(5): 486-92. 